\title{
Power and efficiency performances of a micro thermal Brownian heat engine with and without external forces
}

\author{
Zemin Ding, Lingen Chen*, and Fengrui Sun \\ Postgraduate School, Naval University of Engineering, Wuhan 430033, P.R. China
}

(Received on 4 August, 2009)

\begin{abstract}
Power and efficiency performances of a thermal Brownian heat engine, which consists of Brownian particles moving in a periodic sawtooth potential with and without external forces and contacting with alternating hot and cold reservoirs along the space coordinate, are studied in this paper. The performance characteristics are obtained by numerical calculations. It is shown that due to the heat flow via the change of kinetic energy of the particles, the Brownian heat engine is always irreversible and the efficiency can never approach the Carnot efficiency. The influences of the operation parameters, i.e. barrier height of the potential, asymmetry of the potential and temperature ratio of the heat reservoirs on the power output, the efficiency and the current performances of the Brownian heat engine are investigated in detail by numerical analysis. When there is no external force, the power output versus efficiency characteristic curves are closed loop-shaped ones, which are similar to those of real conventional irreversible heat engines; whereas when the external force is considered, the power output versus efficiency characteristic curves of the heat engine changed into open loop-shaped ones. Furthermore, the limited regions of the external force and barrier height of the potential are explored by analyzing the current property of the model. It is shown that by reasonable choice of the parameters, the Brownian heat engine can be controlled to operate in the optimal regimes.
\end{abstract}

Keywords: Brownian heat engine, power output, efficiency, current, performance characteristics.

\begin{tabular}{|c|c|}
\hline \multicolumn{2}{|c|}{ Nomenclature } \\
\hline A & a defined function \\
\hline$a$ & a defined function \\
\hline B & a defined function \\
\hline$b$ & a defined function \\
\hline$F$ & external force \\
\hline$f$ & scaled external force \\
\hline G & a defined function \\
\hline$H$ & a defined function \\
\hline I & a defined function \\
\hline$J$ & steady state current \\
\hline$k_{\text {B }}$ & Boltzmann's constant \\
\hline$L$ & period length of the potential \\
\hline$L_{1}, L_{2}$ & length of the left and right part of the potential \\
\hline$P$ & power output (W) \\
\hline$\dot{Q}$ & rate of heat flow (W) \\
\hline$T$ & temperature $(\mathrm{K})$ \\
\hline$U$ & scaled barrier height of the potential \\
\hline$U_{0}$ & barrier height of the potential \\
\hline$v$ & drift velocity of the particle \\
\hline$x$ & horizontal axis of the coordinate \\
\hline
\end{tabular}

*Electronic address: lgchenna@yahoo.com and lingenchen@hotmail.com

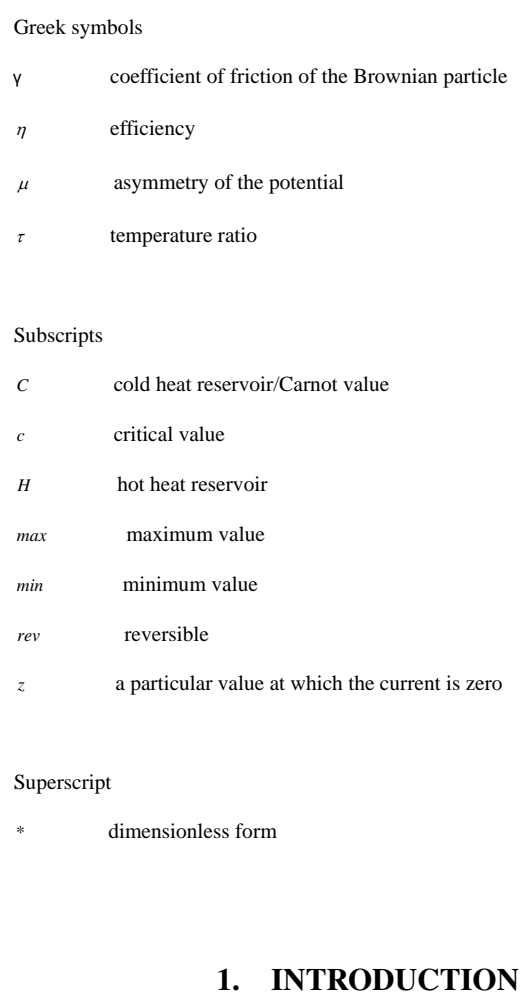

Recently, there have been many interests in the study of Brownian heat engines. One motivation is the need to develop miniaturized devices in order to utilize energy resources at the microscopic scale [1-3]; and another motivation is the desire in understanding the molecular motors in bioengineering and nanotechnology $[4,5]$.

Brownian heat engines are usually spatially asymmetric but periodic structure where the transport of Brownian particles is driven by certain nonequilibrium processes [6-9]. The model of Brownian motor was first presented by Feynman [10] as a thermal ratchet. It is a machine that can rectify thermal fluctuation to produce a directed current. Actu- 


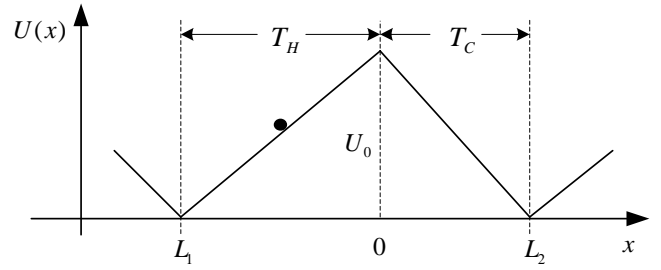

FIG. 1: Schematic diagram of the thermally driven Brownian heat engine.

ally, besides the thermal fluctuation, the nonequilibrium driving force of the Brownian motors can be various. Typical examples are external modulation of an underlying potential [11-13], external force [14-16], chemical potential differences $[6,17]$, temperature differences $[7,8,18-20]$ and so on. According to the nonequilibrium driving force of the Brownian motors, Parrondo and de Cisneros [21] have distinguished the Brownian motors mainly into three classes: the forced ratchet (motor), the chemical motors and the thermal motors. The Brownian heat engine that will be studied in this paper just belongs to the thermal Brownian motors.

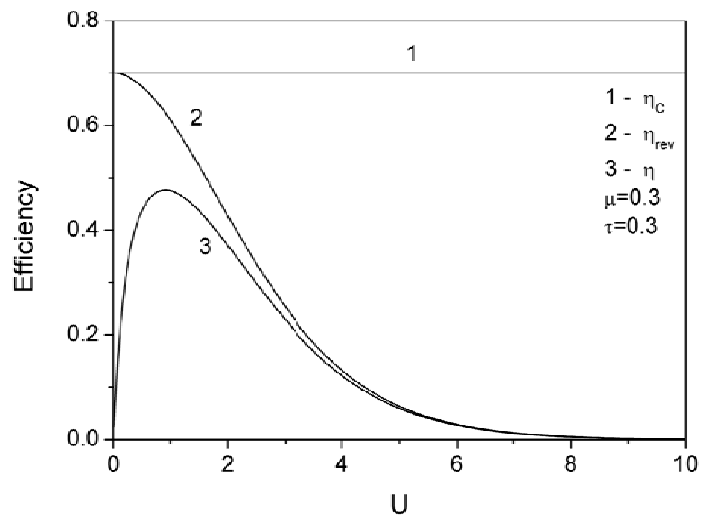

FIG. 2: Characteristic curves of $\eta, \eta_{\text {rev }}$ and Carnot efficiency $\left(\eta_{C}\right)$ versus barrier height $U$, for given $\mu=0.3$ and $\tau=0.3$.

The model of thermal Brownian motor working due to nonuniform temperature was first studied by Büttiker [18], van Kampen [19] and Landauer [20]. And ever since then, the Brownian heat engine model driven by a contact with the heat reservoirs at different temperatures has been the subject of some authors [22-26] and many meaningful results have been obtained. As to the studies of Brownian motors, most have been on the velocity properties of the transport of the Brownian particles; whereas another important aspect is their thermodynamic properties [3,8,27-28]. Derényi et al. [29] gave a definition of generalized efficiency of the microscopic engines and analyzed its application to a Brownian heat engine. Meanwhile, some researchers $[8,9,12,23,28,30,31]$ had investigated the efficiency performance of Brownian heat engines. In Matsuo and Sasa's work [32], it was shown that the efficiency of the Brownian heat engine can attained Carnot efficiency at quasistatic limit. However, this is the case in which the irreversible heat flow via the kinetic energy of the particles $[30,33]$ is neglected. If the heat flow via the kinetic energy is taken into account, the Brownian heat engine can never approach Carnot efficiency [34-37], even at quasistatic limit.

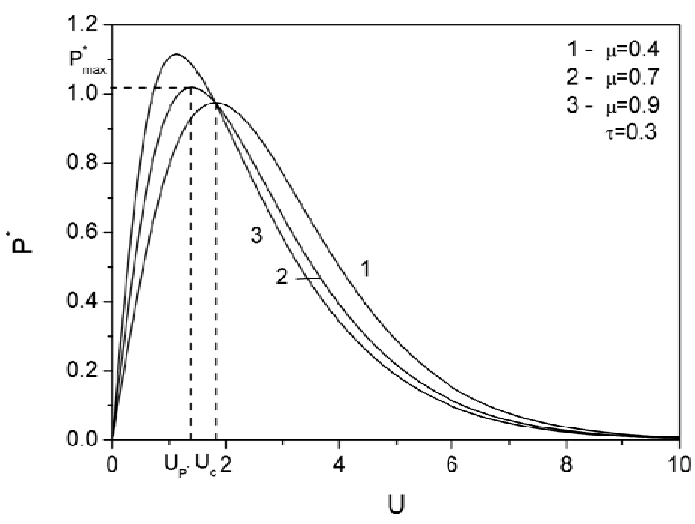

(a)

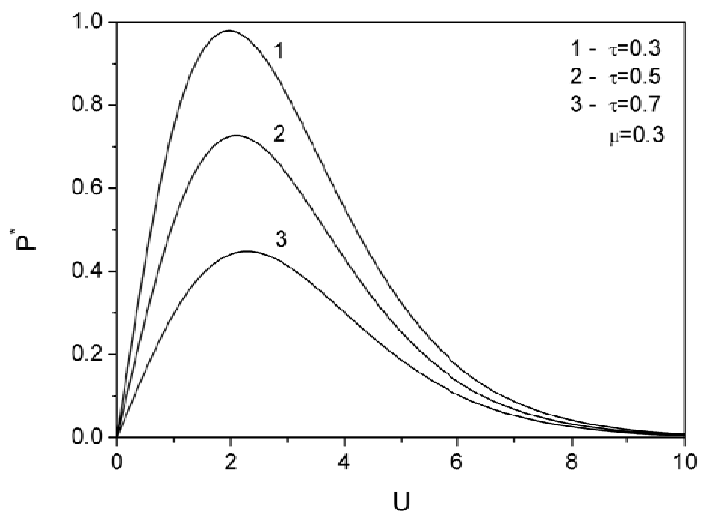

(b)

FIG. 3: Influences of $\mu$ and $\tau$ on the power output $\left(P^{*}\right)$ versus barrier height $(U)$ characteristic.

Without considering the heat flow via the kinetic energy, Asfaw and Bekele [23, 31] studied the efficiency and coefficient of performance (COP) of a Brownian motor model driven by a contact with heat reservoirs at different temperatures which could act as a heat engine or refrigerator. While Ai et al. [25,34], Zhang et al. [35], Lin and Chen [36] investigated the performances of the similar Brownian micro heat engine and refrigerator models by taking into account the influence of the heat flow via the kinetic energy and got many different results. Furthermore, in a recent work, Asfaw [38] presented a Brownian engine model which considered the viscous drag force of the particles and the heat flows via both the potential and kinetic energies. The effects of subdividing the ratchet potential on the velocity and efficiency performances are investigated. The model can act as a heat engine or a refrigerator and it is shown that the velocity and efficiency (or COP) can be maximized when the sawtooth potential is subdivided into series of smaller con- 
nected barrier series. However, the focus of this work [38] was on the velocity and efficiency performances while the thermodynamic properties of the heat engine model, such as the power versus efficiency characteristic and the influences of the operation parameters had not been extensively investigated. Thus, a further step made in this paper is to analyze the thermodynamic performances of such a Brownian heat engine model with and without external forces extensively. The general expressions of the power and efficiency as functions of the major parameters that characterizing the engine are derived. The power versus efficiency characteristic and the influences of external force and other operation parameters are analyzed in detail by numerical calculations. Moreover, the optimal regions of power and efficiency, as well as the limited regions of the external force and barrier height of the potential are determined. It is found that, if these parameters are properly chosen, the Brownian heat engine can operate in the optimum regimes.

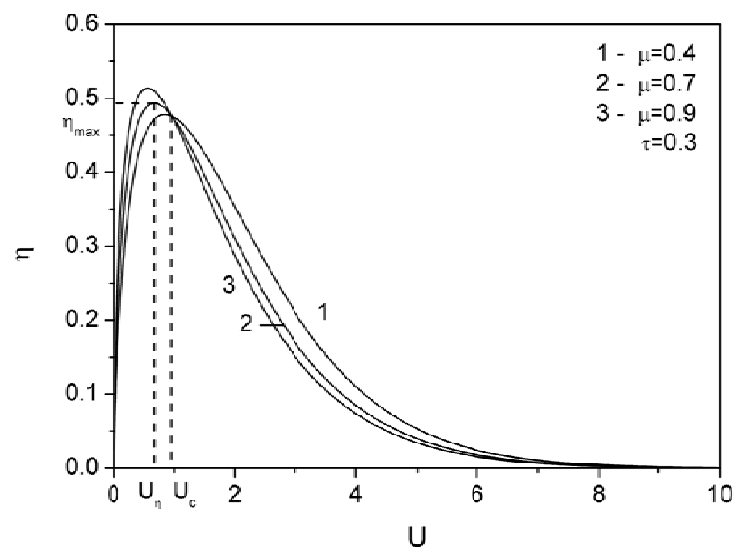

(a)

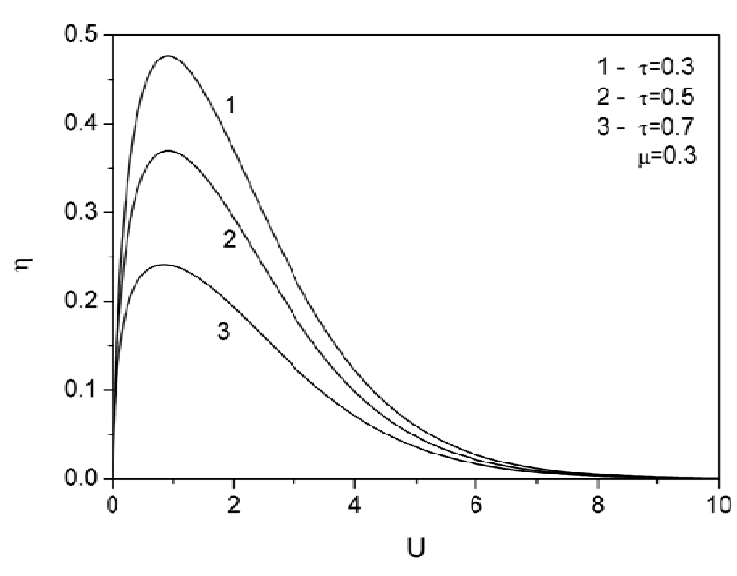

(b)

FIG. 4: Influences of $\mu$ and $\tau$ on the efficiency ( $\eta$ ) versus barrier height $(U)$ characteristic.

\section{MODEL OF THE BROWNIAN HEAT ENGINE}

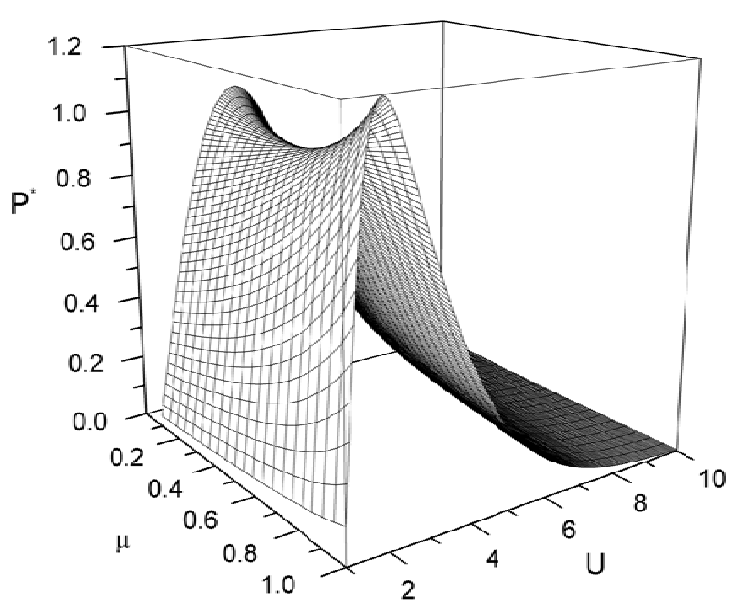

FIG. 5: Three dimensional diagram of power output $\left(P^{*}\right)$ versus $\mu$ and $U$ for given $\tau=0.3$.

Figure 1 shows the schematic diagram of the thermally driven Brownian heat engine. In the heat engine system, the Brownian particle which is placed in a periodic sawtooth potential moves in a viscous medium. The medium is alternatively in contact with the hot (the temperature is $T_{H}$ ) and cold (the temperature is $T_{C}$ ) heat reservoirs along the space coordinate. The sawtooth potential is described by

$$
U(x)= \begin{cases}U_{0}\left(1+x / L_{1}\right), & \text { if }-L_{1}<x \leq 0 \\ U_{0}\left(1-x / L_{2}\right), & \text { if } 0<x \leq L_{2}\end{cases}
$$

where $x$ is the horizontal axis of the coordinate; $L_{1}$ and $L_{2}$, are respectively, the widths of the left and right part of the ratchet; $L=L_{1}+L_{2}$ is the period length and $U_{0}$ is the barrier height of the potential.

The Brownian particle will attain a directed motion when it is exposed to the potential coupled with spatially periodic temperature field. For such a system, according to Refs. [23, 26 , if the external force $F$ is considered, the general expression for the steady state current $J$ for the Brownian particle in any periodic potential is given by

$$
J=\frac{-I}{G_{1} G_{2}+H I}
$$

where $I, G_{1}, G_{2}$ and $H$ are

$$
I=e^{a-b}-1
$$

$$
\begin{aligned}
G_{1} & =\frac{L_{1}}{a T_{H}}\left(1-e^{-a}\right)+\frac{L_{2}}{b T_{C}} e^{-a}\left(e^{b}-1\right), G_{2} \\
& =\frac{\gamma L_{1}}{a}\left(e^{a}-1\right)+\frac{\gamma L_{2}}{b} e^{a}\left(1-e^{-b}\right)
\end{aligned}
$$

$$
H=A+B+C
$$




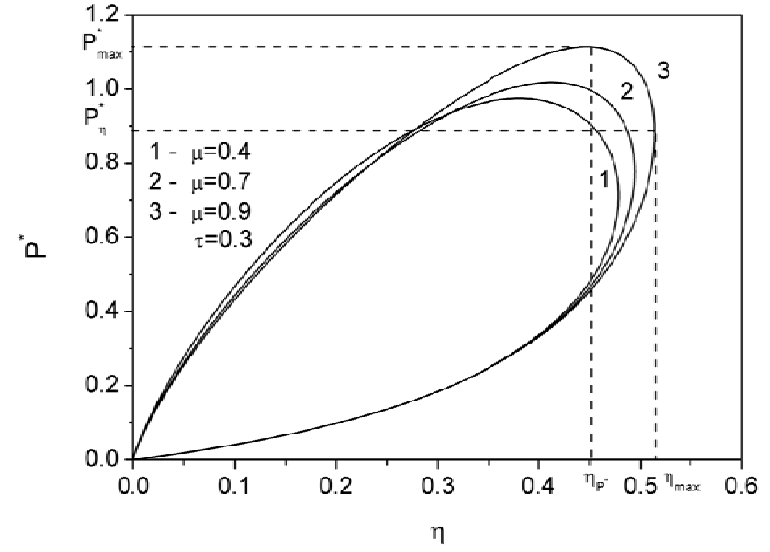

(a)

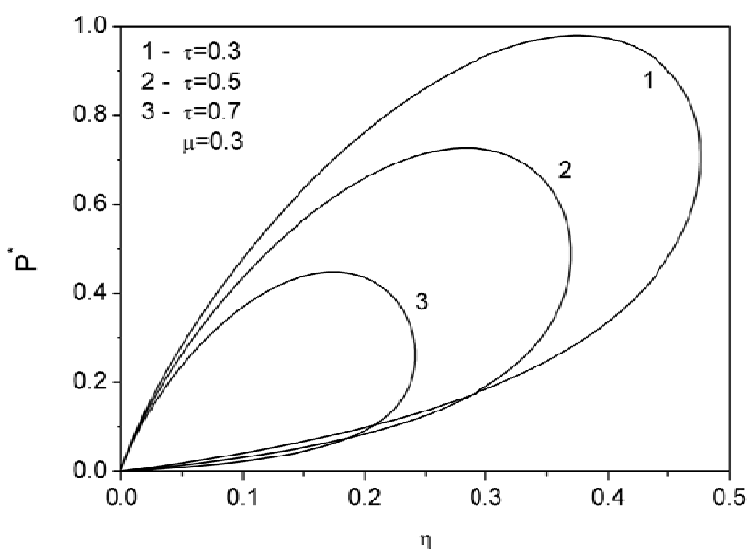

(b)

FIG. 6: Influences of $\mu$ and $\tau$ on the power output $\left(P^{*}\right)$ versus efficiency $(\eta)$ characteristic.

where $\gamma$ is the coefficient of friction of the Brownian particle, and

$$
\begin{aligned}
A & =\frac{\gamma}{T_{H}}\left(\frac{L_{1}}{a}\right)^{2}\left(e^{-a}+a-1\right), \\
B & =\frac{\gamma L_{1} L_{2}}{a b T_{C}}\left(1-e^{-a}\right)\left(e^{b}-1\right), C=\frac{\gamma}{T_{C}}\left(\frac{L_{2}}{b}\right)^{2}\left(e^{b}-b-1\right) \\
a & =\left(U_{0}+F L_{1}\right) / T_{H}, b=\left(U_{0}-F L_{2}\right) / T_{C}
\end{aligned}
$$

If $F=0$, equation (2) becomes the current when there is no external force. The drift velocity $v$ of the particle is associated to the steady state current $J$ and it is given by $v=J L$.

If the system works as a heat engine, the net flux of the particle is from hot reservoir to the cold reservoir. Thus, when the particle moves in the hot region of the ratchet, it must absorb energy in order to climb up the potential and to overcome the external force $(F)$ and the viscous drag force $(\gamma v)$; when it moves in the cold region, the particle will release energy as it moves down the potential hill while at the same time absorb energy to overcome the external force and the viscous drag force. Considering the heat flow $\left(k_{B}\left(T_{H}-T_{L}\right) / 2\right)$ via the kinetic energy of the particles [30,

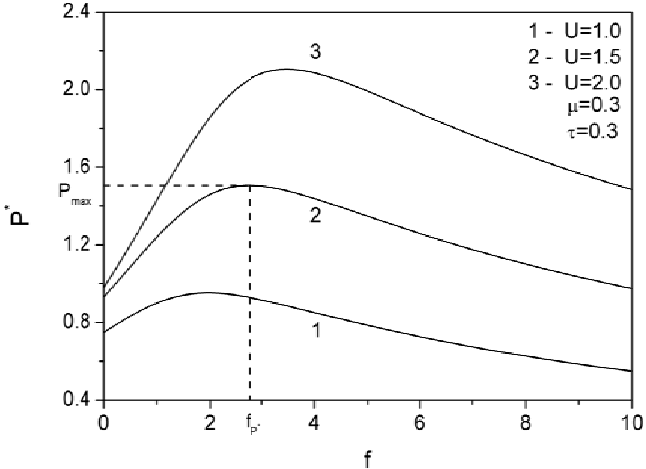

(a)

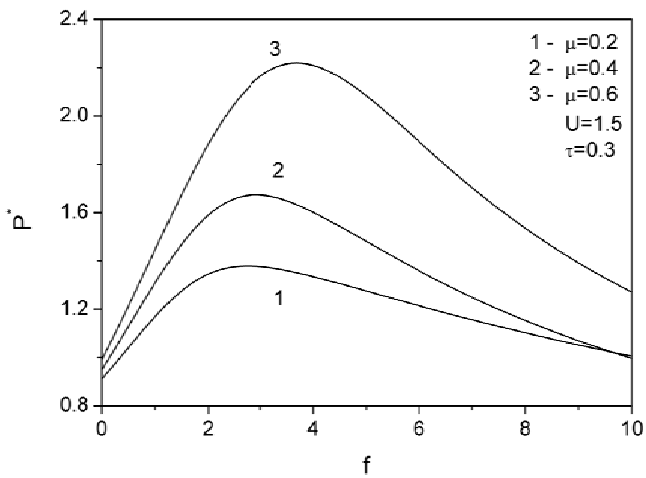

(b)

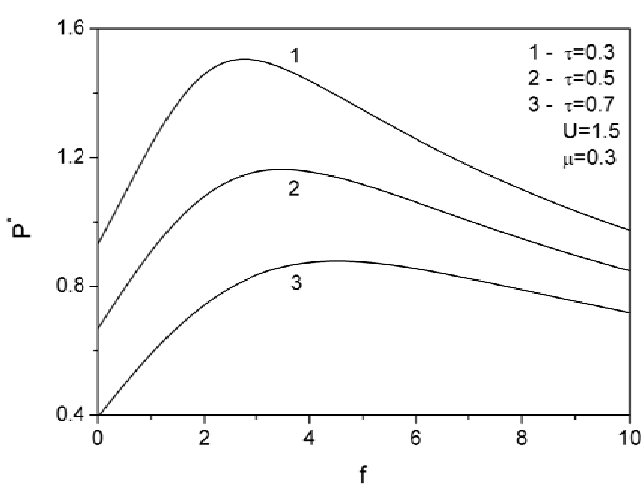

(c)

FIG. 7: Influences of $U, \mu$ and $\tau$ on the power output $\left(P^{*}\right)$ versus external force $(f)$ characteristic.

33], the rates of total heat absorbed from the hot reservoir $\left(\dot{Q}_{H}\right)$ and released to the cold reservoir $\left(\dot{Q}_{C}\right)$ will be [38]

$$
\begin{aligned}
& \dot{Q}_{H}=U_{0}+(F+\gamma v) L_{1}+k_{B}\left(T_{H}-T_{C}\right) / 2 \\
& \dot{Q}_{C}=U_{0}-(F+\gamma v) L_{2}+k_{B}\left(T_{H}-T_{C}\right) / 2
\end{aligned}
$$

where $k_{B}$ is the Boltzmann's constant. 


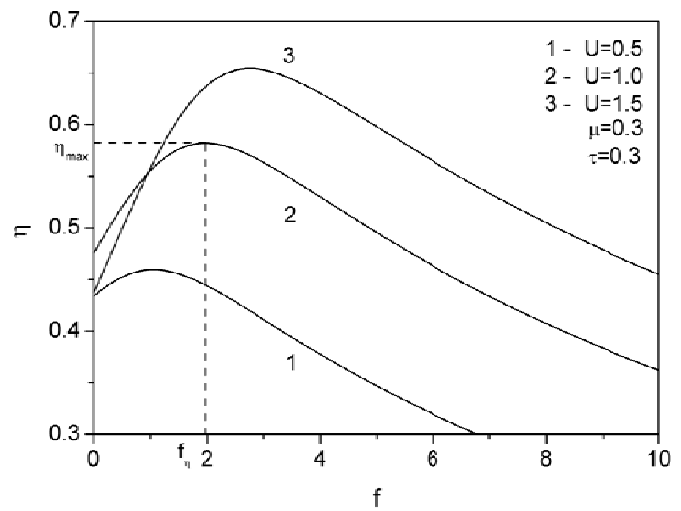

(a)

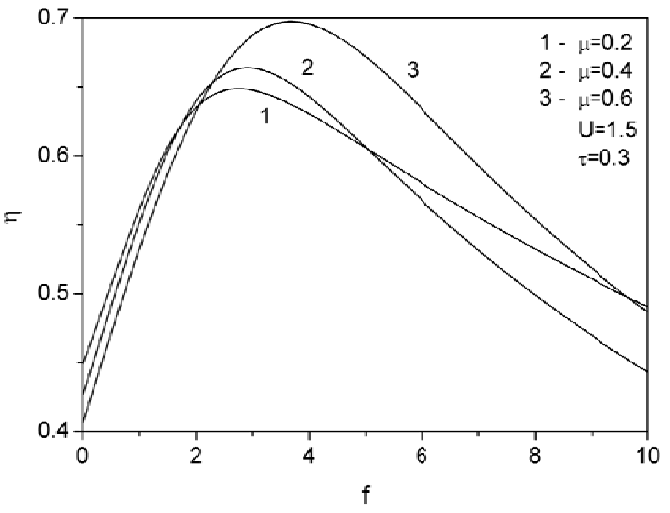

(b)

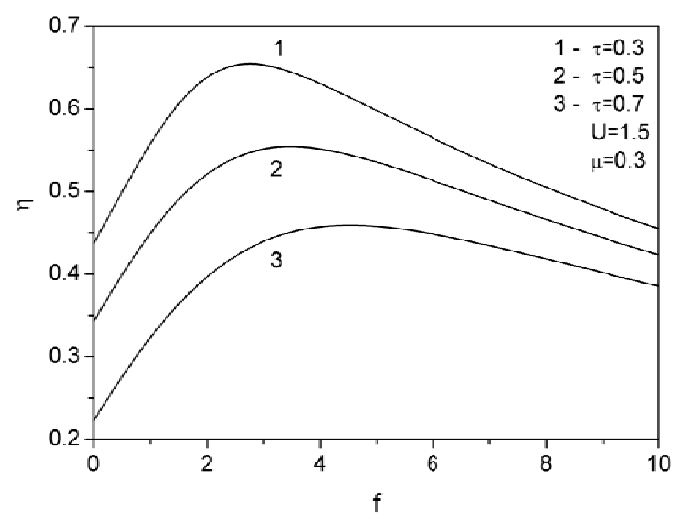

(c)

FIG. 8: Influences of $U, \mu$ and $\tau$ on efficiency $(\eta)$ versus external force $(f)$ characteristic.

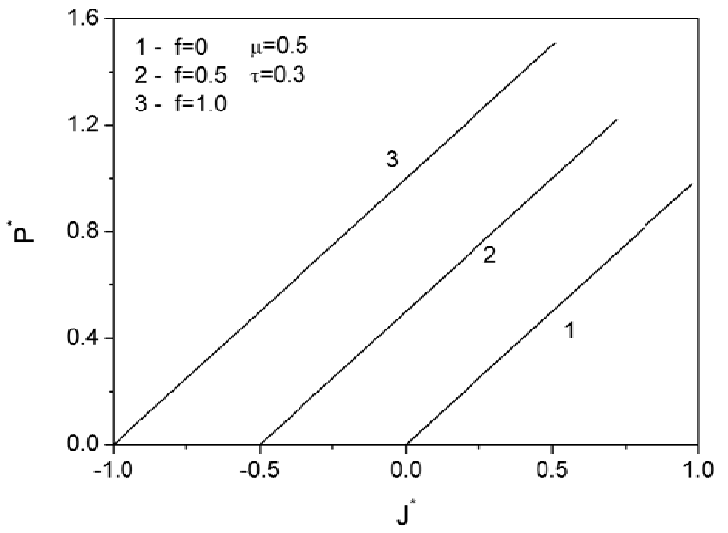

FIG. 9: Influences of external force $(f)$ on the power output $\left(P^{*}\right)$ versus the current $\left(J^{*}\right)$ characteristic, for given $\mu=0.5$ and $\tau=0.3$.

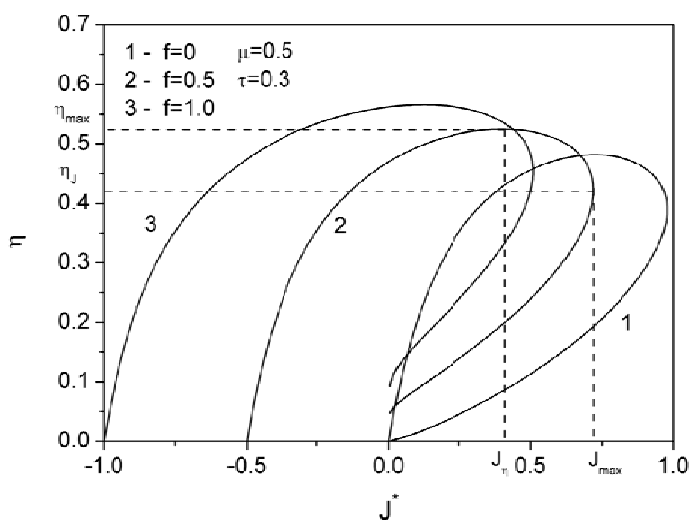

FIG. 10:

Influences of external force $(f)$ on efficiency $(\eta)$ versus the current $\left(J^{*}\right)$ characteristic, for given $\mu=0.5$ and $\tau=0.3$.

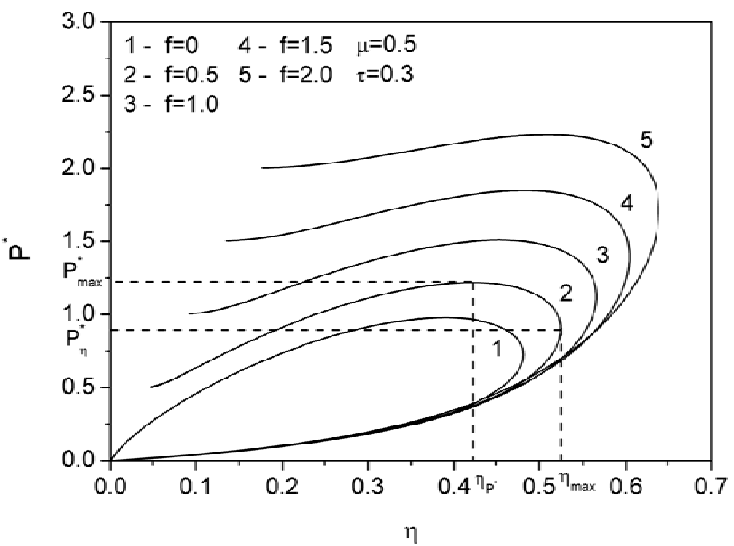

FIG. 11: Influences of external force $(f)$ on the power output $\left(P^{*}\right)$ versus efficiency $(\eta)$ characteristic, for given $\mu=0.5$ and $\tau=0.3$. 
The power output $(P)$ and the efficiency $(\eta)$ of the Brownian heat engine can be expressed as

$$
\begin{aligned}
& P=\dot{Q}_{H}-\dot{Q}_{C}=(F+\gamma v)\left(L_{1}+L_{2}\right)=L(F+\gamma v) \\
& \eta=\frac{P}{\dot{Q}_{H}}=\frac{L(F+\gamma v)}{U_{0}+(F+\gamma v) L_{1}+k_{B}\left(T_{H}-T_{C}\right) / 2}
\end{aligned}
$$

If the heat flow via the kinetic energy is ignored, the reversible efficiency of the Brownian heat engine $\left(\eta_{\text {rev }}\right)$ is given by

$$
\eta_{\text {rev }}=\frac{L(F+\gamma v)}{U_{0}+(F+\gamma v) L_{1}}
$$

For the convenience of the following discussion, equations (2) and (7) can be rewritten into dimensionless forms

$$
\begin{aligned}
& J^{*}=J /\left[T_{H} /\left(\gamma L^{2}\right)\right] \\
& P^{*}=P /\left(k_{B} T_{H}\right)
\end{aligned}
$$

As to the heat engine model, we introduce some scaled parameters, i.e. the scaled barrier height of potential $U=$ $U_{0} /\left(k_{B} T_{H}\right)$, the scaled external force $f=F L /\left(k_{B} T_{H}\right)$, the asymmetry of the potential $\mu=L_{1} / L$ and the temperature ratio of heat reservoirs $\tau=T_{C} / T_{H}$. Equations (8) - (11) can be expressed as functions of the parameters $U, f, \mu$ and $\tau$. For simplicity, the Boltzmann's constant $k_{B}$ is taken to be unity. In the following two sections, the power versus efficiency performance and the influences of these parameters will be investigated with and without external force, respectively, in detail.

\section{POWER VERSUS EFFICIENCY CHARACTERISTIC WITHOUT EXTERNAL FORCE}

In the absence of the external force, i.e. $F=0$, using equations (8) and (9), one can obtain the characteristic curves of $\eta, \eta_{\text {rev }}$ and Carnot efficiency $\eta_{C}\left(\eta_{C}=1-\tau\right)$ versus the barrier height $U$, as shown in Figure 2. It can be seen that $\eta_{\text {rev }}$ is a monotonic decreasing function of $U$. When the heat engine approaches the quasistatic limit, i.e. $U \rightarrow 0$, $\eta_{\text {rev }}$ will attain the Carnot efficiency $\left(\eta_{C}\right)$. It is also clear that, the value of $\eta$ is always smaller than $\eta_{\text {rev }}$ and thus it can never approach Carnot efficiency $\left(\eta_{C}\right)$.

Using equations (8) and (11) and the condition $F=0$, for given values of $\mu$ and $\tau$, the characteristic curves of power output $\left(P^{*}\right)$ and efficiency $(\eta)$ versus barrier height $(U)$ can be plotted as shown in Figures 3 and 4 . It can be seen that the curves of $P^{*}$ and $\eta$ versus $U$ are all parabolic-like ones, and there exist the optimal barrier height $U_{P^{*}}$ and $U_{\eta}$ which lead to the maximum power output $\left(P_{\max }^{*}\right)$ and maximum efficiency $\left(\eta_{\max }\right)$, respectively. It is shown in Figures 3 (a) and 4 (a) that, for given values of $\tau$, the values of $U_{P^{*}}$ and $U_{\eta}$ will decrease with the increase of $\mu$; there exists the critical value of barrier height $\left(U_{c}\right)$ for the characteristic curve; if $U<U_{c}$, $P^{*}$ and $\mu$ will increase with the increase of ; if $U>U_{c}, P^{*}$ and $\eta$ will decrease with the increase of $\mu$. While for given values of $\mu$, as shown in Figure 3 (b) and 4 (b), the values of $U_{\eta}, P_{\max }^{*}$ and $\eta_{\max }$ will decrease whereas $U_{P^{*}}$ will increase with the increase of $\tau$.
Figure 5 shows the three dimensional diagram of power output $\left(P^{*}\right)$ versus barrier height $(U)$ and symmetry of the potential $(\mu)$ with $\tau=0.3$. It can be seen that, for given values of $\mu$, the curves of $P^{*}$ versus $U$ are parabolic-like ones; while for given $U$, the curves of $P^{*}$ versus $\mu$ are monotonic ones. It is found that the maximum power output $\left(P_{\text {max }}^{*}\right)$ first decreases and then increases with the increase of $\mu$. Similarly, using equation (8) and the condition $F=0$, one can get the three dimensional diagram of efficiency $(\eta)$ versus $U$ and $\mu$ with $\tau=0.3$. Its shape is similar to Figure 5 and the maximum efficiency $\eta_{\text {max }}$ also first decreases and then increases with the increase of $\mu$.

Using equations (8) and (11) and the condition $F=0$, one can obtain the characteristic curves of power output $\left(P^{*}\right)$ versus efficiency $(\eta)$ as shown in Figure 6. Similar to the characteristics of the real conventional irreversible heat engines [39-43], the curves of $P^{*}$ versus $\eta$ are all closed loop-shaped ones and there exist two important points of states: the maximum power point $\left(\eta_{P^{*}}, P_{\text {max }}^{*}\right)$ and the maximum efficiency point $\left(\eta_{\max }, P_{\eta}^{*}\right)$. When the Brownian heat engine is operating in the regions $U \leq U_{\eta}$ and $U \geq U_{P^{*}}$, the power output will increase with the increase of efficiency. When the Brownian heat engine is operating in the region $U_{\eta}<U<U_{P^{*}}$, the power output will decrease with the increase of efficiency. Usually, for a heat engine, one always wants to obtain the power and efficiency as large as possible for a given set of other parameters. Thus, it is more reasonable for the Brownian heat engine to operate in the region $U_{\eta}<U<U_{P^{*}}$, which will lead to the optimal regions of power and efficiency, i.e.

$$
P_{\eta}^{*}<P^{*}<P_{\max }^{*} \quad, \quad \eta_{P^{*}}<\eta<\eta_{\max }
$$

And the optimal barrier height of the potential should be located in the region

$$
k_{B} T_{H} U_{\eta}<U_{0}<k_{B} T_{H} U_{P^{*}}
$$

\section{POWER VERSUS EFFICIENCY CHARACTERISTIC WITH EXTERNAL FORCE}

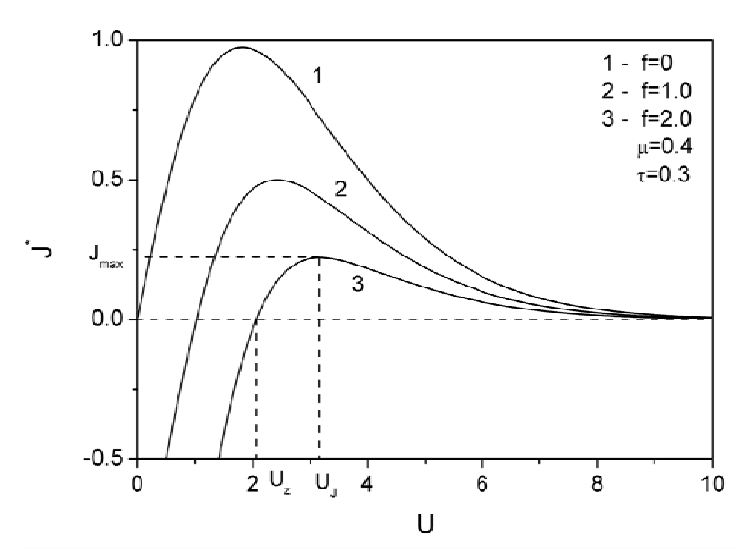

FIG. 12: Characteristic curves of current $\left(J^{*}\right)$ versus barrier height $(U)$ for given $\mu=0.4$ and $\tau=0.3$. 
Using equations (8) and (11), the characteristic curves of power output $\left(P^{*}\right)$ and efficiency $(\eta)$ versus external force $(f)$ and the influences of $U, \mu$ and $\tau$ can be plotted as shown in Figures 7 and 8. It is shown that the curves of $P^{*}$ and $\eta$ versus $f$ are all parabolic-like ones, and there exist optimal external forces $f_{P^{*}}$ and $f_{\eta}$ which lead to the maximum power output $\left(P_{\text {max }}^{*}\right)$ and the maximum efficiency $\left(\eta_{\max }\right)$. For given values of $\mu$ and $\tau$, the values of $P_{\max }^{*}, \eta_{\max }, f_{P^{*}}$ and $f_{\eta}$ will increase with the increase of $U$. For given values of $U$ and $\tau$, the values of $P_{\max }^{*}, \eta_{\max }, f_{P^{*}}$ and $f_{\eta}$ will increase with the increase of $\mu$; and one can see that the influence of $\mu$ here is quite different from the case that there is no external force, where $P_{\max }^{*}$ and $\eta_{\max }$ will first decrease and then increase with the increase of $\mu$, as shown the three dimensional diagram in Figure 5. For given values of $\mu$ and $U$, the values of $P_{\max }^{*}$ and $\eta_{\max }$ will decrease while $f_{P^{*}}$ and $f_{\eta}$ will increase with the increase of $\tau$.

Combining equations (8), (10) with equation (11), the curves of power output $\left(P^{*}\right)$ and efficiency $(\eta)$ as functions of the current $\left(J^{*}\right)$ can be plotted as shown in Figures 9 and 10. It can be seen from Figure 9 that the power output is a monotonic increasing function of current $J^{*}$. While the curves of efficiency versus current are loop-shaped ones, as shown in Figure 10. There exist the maximum efficiency $\eta_{\max }$ and the corresponding current $J_{\eta}^{*}$, as well as the maximum current $J_{\max }^{*}$ and the corresponding efficiency $\eta_{J^{*}}$.

Combining equations (8) with (11), the influence of the external force $(f)$ on the power output $\left(P^{*}\right)$ versus efficiency $(\eta)$ characteristics can be shown in Figure 11. The influences of the external force are obvious: when there is no external force, i.e. $f=0$, the curves of power output versus efficiency are closed loop-shaped ones, as shown by curve 1 in Figure 11; when the external force is applied on the particle, i.e. $f=0.5, f=1.0, f=1.5$ or $f=2.0$, the curves of power output versus efficiency are open loop-shaped ones, as shown by curves 2-5 in Figure 11. There also exist the maximum power $P_{\max }^{*}$ and the corresponding efficiency $\eta_{P^{*}}$, as well as the maximum efficiency $\eta_{\max }$ and the corresponding power output $P_{\eta}^{*}$. The performance of the Brownian heat engine depends greatly on the parameters $f, U, \mu$ and $\tau$; if the values of the parameters are properly chosen, the Brownian heat engine can operate in the optimal regimes.

\section{THE LIMITED REGIONS OF THE EXTERNAL FORCE AND BARRIER HEIGHT OF THE POTENTIAL}

Using equation (10), for given values of $\mu$ and $\tau$, i.e. $\mu=0.4$ and $\tau=0.3$, the characteristic curves of current $\left(J^{*}\right)$ versus barrier height $(U)$ and the influence of external force $(f)$ can be plotted as shown in Figure 12. The curves of $J^{*}$ versus $U$ are parabolic-like ones. When there is no external force $(f=0)$, the current is always higher than zero. This indicates that, without external force, the particle always moves from the hot reservoir to the cold reservoir. When the external force is considered, there exists a critical value of the barrier height $\left(U_{z}\right)$ at which the current changes its direction. If $U<U_{z}$, the current is less than zero, which means that the engine model works as a refrigerator. If $U>U_{z}$, the current is higher than zero, which means that the engine model works as a heat engine. Thus, if external force is con-

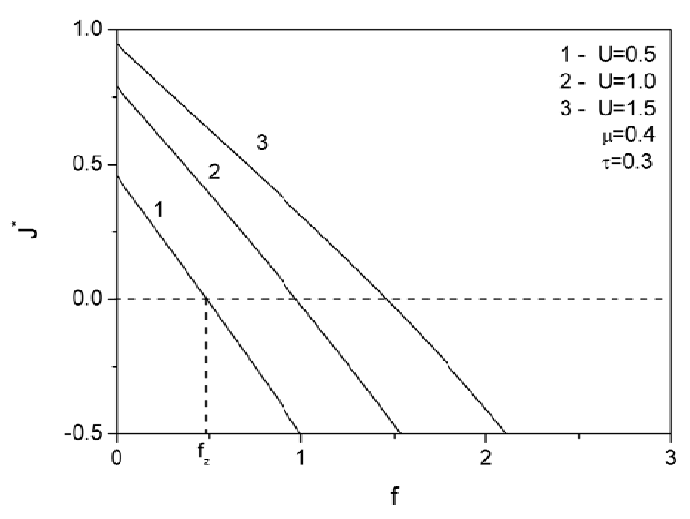

FIG. 13: Characteristic curves of current $\left(J^{*}\right)$ versus external force ( $f$ ) for given $\mu=0.4$ and $\tau=0.3$.

sidered, for the barrier height $\left(U_{0}\right)$, only when the condition that

$$
U_{0}>U_{z} k_{B} T_{H}
$$

is satisfied, the engine model can work as a two-reservoir heat engine. When the engine model works as a heat engine, there exists the optimum barrier height $U_{J^{*}}$ which leads to the maximum current $J_{\max }^{*}$. From Figure 9, one can see that the power output is a monotonic increasing function of the current $J^{*}$. Thus, the optimum barrier height $U_{J^{*}}$ in Figure 12 which corresponds to the maximum current also leads to the maximum power output of the heat engine. With the increase of $f, J_{\max }^{*}$ will decrease while $U_{J^{*}}$ will increase. This is because that the direction of external force is opposite to the movement of the particle, it will hinder the transport of the particle. So the larger the $f$, the less the $J_{\max }^{*}$. Consequently, higher barrier height is needed for the heat engine in order to get the maximum current.

Similarly, using equation (10), for given values of $\mu$ and $\tau$, i.e. $\mu=0.4$ and $\tau=0.3$, the characteristic curves of current $\left(J^{*}\right)$ versus external force $(f)$ and the influence of barrier height $(U)$ can be plotted as shown in Figure 13. It is shown that the current is a monotonic decreasing function of $f$. There exists a particular external force $\left(f_{z}\right)$ at which the current changes its direction. When $0 \leq f<f_{z}$, the current is higher than zero, which means that the engine model works as a heat engine. When $f>f_{z}$, the current is less than zero, which means that the engine model works as a refrigerator. Thus, for the external force $(F)$, only when the condition that

$$
0 \leq F<f_{z} k_{B} T_{H} L
$$

is satisfied, the engine model can work as a two-reservoir heat engine.

Equation (14) is the limited region of barrier height for the Brownian motor to work as a heat engine when there is no external force. Combining equation (14) with (15), one can found that only when the external force and barrier height of the potential are located in the regions $0<F<f_{z} k_{B} T_{H} L$, $U_{0}>U_{z} k_{B} T_{H}$ or $F=0, U_{0}>0$, the Brownian motor can operate as a two-reservoir heat engine. 


\section{CONCLUSIONS}

In the present work, the thermodynamic performance of a thermally driven Brownian heat engine is studied. The heat engine consists of Brownian particles moving in a periodic sawtooth potential with and without external forces, where the viscous medium is alternately in contact with the hot and cold reservoirs along the space coordinate. The performance characteristics are analyzed by numerical calculations. It is shown that the Brownian heat engine is always irreversible due to the heat flow via the change of kinetic energy of the particle, and the efficiency can never approach the Carnot efficiency, even in the quasistatic limit. Numerical analysis has shown that the operation parameters, i.e. barrier height of the potential, asymmetry of the potential and temperature ratio of the heat reservoirs have great influences on the power output, efficiency and current performances of the Brownian heat engine. It is found that when there is no external force, the power output versus efficiency characteristic curves are closed loop-shaped ones, which are similar to the characteristics of real conventional irreversible heat engines; whereas when the external force is applied on the particle, the power output versus efficiency characteris- tic curves of the heat engine changed into open loop-shaped ones. The limited regions of the external force and barrier height of the potential are explored by analyzing the current property of the model. And it is found that only when the external force and barrier height of the potential are located in the region $0<F<f_{z} k_{B} T_{H} L, U_{0}>U_{z} k_{B} T_{H}$ or $F=0, U_{0}>0$, the Brownian motor can operate as a two- reservoir heat engine. Results of numerical calculations show that through the proper choice of the parameters, the Brownian heat engine can be controlled to operate in the optimal regimes. The results obtained herein can provide some guidelines in revealing the general performance characteristics of thermal Brownian heat engines.

\section{Acknowledgments}

This paper is supported by The National Natural Science Foundation of P. R. China (Project No. 10905093), Program for New Century Excellent Talents in University of P. R. China (Project No. NCET-04-1006) and The Foundation for the Author of National Excellent Doctoral Dissertation of P. R. China (Project No. 200136).
[1] P. Reimann, Brownian motors: noisy transport far from equilibrium. Phys. Rep., 2002, 361(2/4): 57-265.

[2] R.D. Astumian, P. Hänggi, Brownian motors. Phys. Today, 2002, 55 (11): 33-39.

[3] C. Van den Broeck, R. Kawai, P. Meurs, Microscopic analysis of a thermal Brownian motor. Phys. Rev. Lett., 2004, 93 (9): 090601.

[4] N. Thomas, R.A. Thornhill, The physics of biological molecular motors. J. Phys. D: Appl. Phys., 1998, 31 (3): 253-266.

[5] M. Schliwa, G. Woehlke, Molecular motors. Nature, 2003, 422(6933): 759-765.

[6] R.D. Astumian, M. Bier, Fluctuation driven ratchets: molecular motors. Phys. Rev. Lett., 1994, 72 (11): 1766-1769.

[7] F. Takagi, T. Hondou, Thermal noise can facilitate energy conversion by a ratchet system. Phys. Rev. E, 1999, 60 (4): 49544957.

[8] K. Sumithra, T. Sintes, Efficiency optimization in forced ratchets due to thermal fluctuations. Physica A, 2001, 297(1/2): 112.

[9] B.Q. Ai, X.J. Wang, G.T. Liu, L.G. Liu. Efficiency optimization in a correlation ratchet with asymmetric unbiased fluctuations. Phys. Rev. E, 2003, 68 (6): 061105.

[10] R.P. Feynman, R.B. Leighton, M. Sands, The Feynman Lectures on Physics. Vol. I Addison-Wesley, Reading, 1963.

[11] L.P. Faucheux, L.S. Bourdieu, P.D. Kaplan, A.J. Libchaber. Optical thermal ratchet. Phys. Rev. Lett., 1995, 74 (99): 15041507.

[12] J.M.R. Parrondo, J.M. Blanco, F.J. Cao, R. Brito. Efficiency of Brownian motors. Europhys. Lett., 1998, 43 (3): 248-254.

[13] L. Dinis, J.M.R. Parrondo, F.J. Cao, Closed-loop strategy with improved current for a flashing ratchet. Europhys. Lett., 2005, 71 (4): 536-541.

[14] J.L. Mateos, Chaotic transport and current reversal in deterministic ratchets. Phys. Rev. Lett., 2000, 84 (2): 258-261.

[15] M.O. Marcelo, Forced thermal ratchets. Phys. Rev. Lett., 1993, 71 (10):1477-1481.

[16] S. Savel'ev, F. Marchesoni, P. Hänggi, F. Nori, Nonlinear sig- nal mixing in a ratchet device. Europhys. Lett., 2004, 67 (2): 179-185.

[17] R.D. Astumian, I. Derényi, A chemically reversible Brownian motor: application to kinesin and Ncd. Biophys J., 1999, 77 (2): 993-1002.

[18] M. Büttiker, Transport as a consequence of state-dependent diffusion. Z. Phys. B, 1987, 68(2/3): 161-167.

[19] N.G. van Kampen, Relative stability in nonuniform temperature. IBM J. Res. Dev., 1988, 32 (1): 107-111.

[20] R. Landauer, Motion out of noisy states. J. Stat. Phys., 1988, 53(1/2): 233-248.

[21] J.M.R. Parrondo, B.J. de Cisneros, Energetics of Brownian motors: a review. Appl. Phys. A, 2002, 75 (2): 179-191.

[22] K. Sekimoto, Kinetic characterization of heat bath and energetics of thermal ratchet. J. Phys. Soc. Jpn., 1997, 66 (5): 1234-1237.

[23] M. Asfaw, M.Bekele, Current, maximum power and optimized efficiency of a Brownian heat engine. Eur. Phys. J. B, 2004, 38 (3): 457-461.

[24] P. Meurs, C. Van den Broeck. Thermal Brownian motor. J. Phys: Condens. Matter, 2005, 17 (47): S3673-S3684.

[25] B.Q. Ai, H.Z. Xie, D.H. Wen, X.M. Liu, L.G. Liu. Heat flow and efficiency in a microscopic engine. Eur. Phys. J. B, 2005, 48 (1): 101-106.

[26] M. Asfaw, M. Bekele, Exploring the operation of a tiny heat engine. Physica A, 2007, 384 (2): 346-358.

[27] R.D. Astumian, Thermodynamics and kinetics of a Brownian motor. Science, 1997, 276(5314): 917-922.

[28] S. Velasco, J.M.M. Roco, A. Medina, A. Calvo Hernández. Feynman's ratchet optimization: maximum power and maximum efficiency regimes. J. Phys. D: Appl. Phys., 2001, 34 (6): 1000-1006.

[29] I. Derényi, M. Bier, R.D. Astumian, Generalized efficiency and its application to microscopic engines. Phys. Rev. Lett., 1999, 83 (5): 903-906.

[30] I. Derényi, R.D. Astumian. Efficiency of Brownian heat engines. Phys. Rev. E, 1999, 59 (6): R6219-R6222. 
[31] M. Asfaw, M. Bekele. Energetics of a simple microscopic heat engine, Phys. Rev. E, 2005, 72 (5): 056109.

[32] M. Matsuo, S. Sasa. Stochastic energetics of non-uniform temperature systems. Physica A, 2000, 276(1/2): 188-200.

[33] T. Hondou,K. Sekimoto, Unattainability of Carnot efficiency in the Brownian heat engine. Phys. Rev. E, 2000, 62 (5): 60216025.

[34] B.Q. Ai, L. Wang, L.G. Liu. Brownian micro-engines and refrigerators in a spatially periodic temperature field: heat flow and performances. Phys. Lett. A, 2006, 352(4/5): 286-290.

[35] Y. Zhang, B. Lin, J. Chen. Performance characteristics of an irreversible thermally driven Brownian microscopic heat engine. Eur. Phys. J. B, 2006, 53 (4): 481-485.

[36] B. Lin, J. Chen, Performance characteristics and parametric optimum criteria of a Brownian micro-refrigerator in a spatially periodic temperature field. J. Phys. A: Math. Theor., 2009, 42 (7): 075006.

[37] R. Benjamin, R. Kawai, Inertial effects in Büttiker-Landauer motor and refrigerator at the overdamped limit. Phys. Rev. E,
2008, 77 (5): 051132.

[38] M. Asfaw, Modeling an efficient Brownian heat engine. Eur. Phys. J. B, 2008, 65 (1): 109-116.

[39] J.M. Gordon, G. Huleihil, General performance characteristics of real heat engine. J. Appl. Phys., 1992, 72 (3): 829-837.

[40] L. Chen, F. Sun, C. Wu, A generalized model of real heat engines and its performance. J. Institute Energy, 1996, 69 (481): 214-222.

[41] L. Chen, F. Sun, C. Wu, Influence of internal heat leak on the power versus efficiency characteristics of heat engines. Energy Convers. Mgmt., 1997, 38 (14): 1501-1507.

[42] L. Chen, F. Sun, C. Wu, Effect of heat transfer law on the performance of a generalized irreversible Carnot engine. J. Phys. D: Appl. Phys., 1999, 32 (2): 99-105.

[43] N. Sanchez Salas, S. Velasco, A. Calvo-Hernandez. Unified working regime of irreversible Carnot-like heat engines with nonlinear heat transfer laws. Energy Convers. Mgnt., 2002, 43 (17): 2341-2348. 